\title{
Moving Vehicle Detection and Recognition Technology based on Artificial Intelligence
}

\author{
Zengfang Shi ${ }^{*}$, Meizhou Liu \\ School of Automotive and Aeronautical Engineering, Henan Polytechnic Institute, Nanyang 473009, \\ China \\ *Corresponding author: Jzshijian@126.com
}

Received: June 4, 2021. Revised: December 12, 2021. Accepted: January 11, 2022. Published: January 13, 2022.

\begin{abstract}
The existing target detection and recognition technology has the problem of fuzzy features of moving vehicles, which leads to poor detection effect. A moving car detection and recognition technology based on artificial intelligence is designed. The point operation is adopted to enhance the high frequency information of the image, increase the image contrast, and delineate the video image tracking target. The motion vector similarity is used to predict the moving target area in the next frame of the image. The texture features of the moving car are extracted by artificial intelligence, and the center moment is calculated by the gray histogram distribution curve, the edge feature extraction algorithm is used to set the detection and recognition mode. Experimental results: under complex conditions, this design technology, compared with the other two kinds of moving vehicle detection and recognition technology, detected three more moving vehicles, which proved that the application prospect of the moving vehicle detection and recognition technology integrated with artificial intelligence is broader.
\end{abstract}

Keywords-artificial intelligence, sports car, detection and identification technology, intelligent transportation, image information, texture features.

\section{INTRODUCTIONS}

$\mathrm{I}^{\mathrm{N}}$ $\mathrm{N}$ the mid-20th century, the intelligent transportation management system has just started and is in its infancy. In the later stage, developed countries led by the United States and Japan began to focus on the intelligent transportation system. At present, the United States, Japan and the European Union have formed their own independent intelligent transportation architecture. As the key technology of the system, that is, the detection and recognition technology of sports vehicles, the United States and Japan have made a lot of improvements. In 1978, the United States first used computer vision technology to detect vehicles, marking a new era of vehicle detection. In the ordinary environment, the technology of vehicle detection and recognition is relatively mature. Vehicle detection and recognition technology in ordinary environment is relatively mature, how to calculate according to motion estimation, Markov random field and other technologies to solve the occlusion problem of moving objects [1], [2]. The research on the detection and recognition technology of moving vehicles in China started in the 1980s, when it was the primary stage of China's economic development. The transportation hardware resources fully met the demand of traffic volume, so China only introduced some related projects from abroad. Intelligent transportation system (ITS) is a frontier topic in the field of transportation in the world. It integrates advanced detection, communication and computer technology into automobile and road to form a road transportation system, with the ultimate goal of safety, smooth flow, low pollution and high efficiency. The system detects, identifies and analyzes the moving objects in the traffic video sequence, especially the moving cars, to help solve the daily management of traffic. The research shows that the intelligent transportation system can greatly improve the traffic capacity and service level of the highway, increase the traffic flow of each lane by 2-3 times per hour, and shorten the driving time by $35 \%-50 \%$. In addition, the intelligent transportation system can greatly improve the safety of highway traffic, reduce the negative impact of drivers' psychology on traffic safety, eliminate human errors, and make it possible to prevent and avoid traffic accidents [3]. Intelligent transportation system relies on sports vehicle target detection and recognition technology, which has had some research results. Hakim and $\mathrm{Zul}$ [4] proposed the discrete wavelet transform and recognition used to focus on the target detection and recognition of motor vehicles. A quantitative analysis of change background statistics on the capability of tracking objects using "mean shift" procedure is present. Change of background statistics assumed changing of mean of brightness and changing noise variance in the scene. Quantitative analysis implies detection error and number of iteration needed for position determination using "mean shift" procedure, to complete the vehicle detection and identification. Chen et al. [5] proposed a fast detection algorithm for Fast RCNN motor vehicles based on spatiotemporal fusion acceleration. The time domain motion characteristics and airspace related characteristics 
of the motor vehicle targets are considered. First, the adaptive dynamic background estimation method is designed based on time domain motion features to estimate background images in real-time, morphological filtering is used to quickly extract suspected target regions based on airspace features; Finally, the Fast RCNN deep learning network is designed based on vgg16 model to accurately distinguish the suspected target area and avoid the full map redundancy.

However, the above target detection and identification technology has the problem of fuzzy motor vehicle characteristics, and the detection effect is not good. To improve the detection effect, this paper proposes an AI-based detection and recognition technique of motion vehicles. Point operation is used to improve the image contrast, to outline the video image tracking target, to predict the motion area of the motion vector, to extract the characteristics of the gray histogram distribution curve, and to determine the vehicle detection and recognition mode using the edge feature extraction algorithm. The experimental results show that the detection and recognition of motor vehicles are better.

As a comprehensive subject of machine intelligence and intelligent machine, artificial intelligence involves information science, psychology, cognitive science, thinking science, biological science and other disciplines. At present, it has been applied in knowledge processing, pattern recognition, machine learning, natural language processing, game theory, automatic theorem proving, automatic programming, expert system, knowledge base, etc. Intelligent robot and other fields have achieved practical results [6]. At present, the research data about the integration of artificial intelligence technology and moving vehicle detection and recognition technology is not very rich, which needs to be further discussed.

\section{RESEARCH ON MOVING VEHICLE DETECTION AND}

\section{RECOGNITION TECHNOLOGY BASED ON ARTIFICIAL}

\section{INTELLIGENCE}

\section{A. High frequency information enhancement of image by point operation}

High frequency information enhancement is a basic image processing technology, which can improve image quality by highlighting some information in the image and suppressing or removing unnecessary information at the same time. For example, enhance the high frequency information of the image to obtain a clearer image of the target contour. If the image is operated point by point, the operation process is specific to each pixel, which is called point operation image enhancement [7], [8]. If the process is in the neighborhood of each pixel, it is called template operation or spatial domain filtering. In image processing, the method based on point operation is a simple and effective method for image enhancement in image space domain [9]. Image enhancement based on point operation mainly includes the following three methods: one is to transform the gray value of each pixel in the original image directly; the second is to transform the original image with the help of gray histogram; the third is the method of transformation by means of a series of operations between images. Gray transformation is generally used to expand the range of gray value of image and increase the contrast of image. On this basis, the gray transformation formula of the image in the pixel is obtained as follows:

$$
\theta=Q \times \beta^{-e}
$$

In Formula (1), $Q$ is the input image, $\beta$ is the output image, and $e$ is the gray transformation coefficient. It is the key to choose the appropriate gray transform function to determine the effect of the image enhancement method. In practice, we can flexibly design the gray transform function according to the needs. Histogram is the statistical result of the number of gray values of each pixel in an image. It can only reflect the gray value distribution of the image, but can not reflect the location of a certain gray value pixel, let alone describe the image completely [10]-[12]. Generally, the abscissa is used to represent the gray value, and the ordinate is used to represent the number of times the gray value appears. If the range of gray value is $[0, p-1]$, the discrete function of histogram can be expressed as:

$$
w\left(\beta_{u}\right)=r_{u}, u=0,1 \cdots, p-1
$$

In Formula (2), $w$ is the discrete function, $r$ is the gray level of the image, $u$ is the number of pixels, $p$ is the total number of gray levels in the image, and $\beta$ is the output image. In practical application, normalized histogram is usually used to analyze the distribution of image gray level, which can be obtained by calculating the probability value of each gray level. On the basis of Formula (2), the expression formula of the probability value of gray level pixels in the image is obtained as follows:

$$
T=\frac{r_{u}}{\varepsilon}(3)
$$

In Formula (3), $r$ is the gray level of the image, $u$ is the number of pixels, and $\varepsilon$ is the gray value after normalization. Different image enhancement methods are used for different images, or several appropriate enhancement algorithms are used for experiments at the same time, from which the method with good visual effect and moderate amount of calculation is selected. Based on the above calculation, the step of point operation to enhance high frequency information of image is completed.

\section{B. Delineation of video image tracking target}

Target tracking means that after target detection and recognition, the target position is determined in each frame of video image by using the target position information, so as to obtain the target trajectory information. Moving object tracking is an important content of moving vehicle detection and recognition technology, and also a research hotspot in the field of computer vision, which plays an important role in improving vehicle driving safety [13]. The target feature information (such as shape, contour and texture features) in the target region is used to search the 
subsequent video image frames, and the region matching the target region is detected, so as to obtain the position of the tracking target [14]. By concatenating the isolated target regions of each frame in time, the target tracking results of video image can be obtained. The specific tracking process is as follows: 1. Detecting the car in the first frame image. Assuming that the calibration vehicle is $\alpha$, the expression formula of vehicle centroid is as follows:

$$
\left\{\begin{array}{c}
\operatorname{Ctr}_{\alpha}=[A, B] \\
A=\sum_{i=1}^{E} A_{i} \\
B=\sum_{i=1}^{E} B_{i}
\end{array}\right.
$$

In Formula (4), $A$ represents the abscissa of the pixel, $B$ represents the ordinate of the pixel, $E$ represents two adjacent pixels, and $i$ represents the pixel of the image car. 2. Detecting the cars in the next frame and calculate their respective area and centroid position. 3. Calculate the distance and area difference between the car and the centroids of each car in the next frame, the expression formula of the distance between vehicle centroids is as follows:

$$
D=\sqrt{\left(A_{i}(E)-\eta\right)^{2}+\left(B_{i}(E)-\eta\right)^{2}}
$$

In Formula (5), $A$ represents the abscissa of the pixel, $B$ represents the ordinate of the pixel, $E$ represents two adjacent pixels, $i$ represents the pixel of the image car, and $\eta$ represents the area of the car. 4. Update the area and centroid position data of the tracked car. Go to step 2 and continue to track subsequent frames. Using the above tracking method, we can not only track one car, but also track multiple cars at the same time. The above tracking method is based on the assumption that because the time interval between two adjacent frames is very short $(0.04$ seconds), the moving distance of the car between the two adjacent frames is small, and the area change rate of the car in the image is small. If the tracked car overlaps with other cars in the next frame and cannot be directly realized by the above method, it is necessary to perform the following segmentation after the first tracking failure and then track [15], [16]. When searching for the target in each frame, because the adjacent frames in the video image have similar motion vectors, the motion vector similarity can be used to predict the region of the moving target in the next frame. Thus, the target search area in the image to be measured is reduced, the amount of computation is reduced, the complexity of the algorithm is reduced, and the real-time performance of tracking is improved. In the case of no prior information, the target detection results of the previous frame can be used when searching the image frame. At the same time, the known information is used to establish the target model, which is matched with the video image to obtain the location information of the target, so as to realize the tracking. Since the tracking object in video image can be regarded as the projection from $3 \mathrm{D}$ world to $2 \mathrm{D}$ plane, the $3 \mathrm{D}$ image of the tracking object is projected into $2 \mathrm{D}$ plane, and the region matching the object model is searched in the image, so as to obtain the position information and motion trajectory of the tracking object [17]. For different types of tracking targets, different tracking methods need to be selected according to the target structure and related parameters. Based on this, the link of delineating video image tracking target is completed.

\section{Texture feature extraction of moving car based on}

\section{Artificial Intelligence}

The special artificial intelligence technology in artificial intelligence technology is mainly used to study one or more special fields and functions, such as computer vision, speech recognition, etc. at present, most of the artificial intelligence belongs to this category. Due to the complexity of color space model, shape and texture feature extraction is used. Texture is one of the inherent characteristics of an image, which is a pattern produced by the transformation of gray level in space in a certain form. Texture is an attribute that represents the surface or structure of a target. Objects in nature have all kinds of textures. These external features can reflect the different internal structure of the target. In daily life and production, the moving vehicle texture characteristics of the target is one of the important basis for us to identify and judge the target. Texture is also a spatial distribution attribute that expresses the gray level of pixels in an image. The inherent attribute of this spatial structure can be expressed by the correlation between neighboring pixels. For an image, texture can be considered as a combination of elements that color combines and weaves with each other according to some inherent law. It is a visual feature that does not depend on color or brightness and reflects the essential phenomenon in the image. This feature reflects the sparsity and smoothness of image distribution [18]. The texture is described by the statistical planning of gray distribution and relation of image by artificial intelligence. This method can effectively reflect the sparse, regular and smooth features of image texture. The main goal of texture feature method based on statistics is to estimate the parameters of stochastic process. Texture is a pattern produced by the transformation of gray level in space in a certain form, some of which have periodicity. Histogram is a powerful tool to describe the gray level distribution of pixels in the image, so it can be used to describe the texture features of the object. The method of using gray histogram to describe texture indirectly is to get the central moment through the distribution curve of gray histogram, the expression formula is as follows:

$$
L=\sum_{n=1}^{n}\left(h_{n}-1\right)^{n} g(h)
$$

In Formula (6), $h$ is the order of the central matrix, $g$ is the image contrast, and $n$ is the histogram skewness. The results of Formula (6) can reflect some characteristics of the boundary distribution of the image. Smoothness is a measure of the relative flatness of texture brightness then the expression formula of image smoothness is as follows: 


$$
K=\frac{1}{(1+\varphi)^{2}}(7)
$$

In Formula (7), $\varphi$ represents the number of pixels in the region. If the gray level of the region is consistent, then $K=1$; If there is a big difference in the gray value of the region, then $K=0$. The main information is extracted from the new mapping space, and the feature description of the original data space is constructed through statistical analysis. The variables of the new mapping space are composed of the linear combination of the original data. Image recognition is essentially the process of classifying the objects in the image [19]-[21]. In order to make a correct decision on the category of the object in the image, it is necessary to separate it from other different categories of images combined with artificial intelligence technology. This requires that the extracted features can not only represent the image well, but also distinguish different types of images. According to the characteristics of the target, it can be divided into two categories: the shape feature describing the shape of the target and the texture feature describing the gray change of the target surface. After obtaining various regions of interest through image segmentation, some shape features can be used to represent the region. These features are usually combined into feature vectors for classification. Based on the above description, the step of extracting texture features of moving car is completed.

\section{Edge feature extraction algorithm setting technology detection and recognition mode}

The edge of an image refers to the part of the image where the brightness changes significantly. The gray image of this area can be regarded as a step, that is, from one gray value to another area with large gray difference. The extraction of image edge is very important for the recognition and understanding of the whole image scene, and it is also an important feature of image segmentation [22], [23]. Edge detection is mainly the measurement of image gray level change, which calculates the gray level change of image pixel in space and time to get the edge curve of moving object. Vehicle detection and recognition sub unit in video image and moving vehicle tracking sub unit in video image. Vehicle detection and recognition is the main way to obtain the motion state, and its output image with recognition results will be used as the input of the moving vehicle tracking sub unit to provide training image for it. In the moving vehicle unit, the output target of the vehicle detection and recognition sub unit is tracked, and the position of the target is determined in each frame of the video, so as to obtain the vehicle trajectory information. The expression formula of gray value vector of reference point pixel is as follows:

$$
\Delta l \times S+G=0(8)
$$

In Formula (8), $l$ is the gradient direction of the image, $S$ is the key frame of the image, and $G$ is the fluctuation range of the pixel value. The edge of the image obtained by edge detection is usually discontinuous, and sometimes there is still a little background, which can not completely segment the target and background. In order to connect the discontinuous target edges and remove the background completely, morphological operation is necessary. Morphological operation can simplify the image data and keep the basic shape of the image unchanged. The basic operations include expansion, corrosion, opening and closing. The first closing operation uses vertical linear structure elements, while the second closing operation uses square structure elements. The parameters of structural elements vary with the illumination conditions and vehicle size. Then the boundary is removed and filled to form a binary image. The essence of image blur is that the image is subject to average or integral operation. In order to make the image clear, it is necessary to carry out inverse operation, such as differential operation for continuous image or differential operation for discrete image. The essence of edge detection is the change of image brightness. The operation of edge detection is to calculate the first derivative or the second derivative based on brightness. Because most of the images contain noise interference, especially in the edge is easy to produce a lot of noise burr, so before doing edge detection, we need to smooth the image noise. The essence of image blur is that the image is subject to average or integral operation. In order to make the image clear, it is necessary to carry out inverse operation, such as differential operation for continuous image or differential operation for discrete image. Compactness is the quantity to describe the compactness of image arrangement. In European space, the most compact region is circle, so compactness is the similarity between region and circle, the expression formula is as follows:

$$
\gamma=\frac{d^{2}}{4 \pi V}
$$

In Formula (9), $d$ is the perimeter of the region boundary, and $V$ is the area of the region boundary. When the region is closer to the circle, the value of $\gamma$ is closer to 1. Because the threshold in edge detection is not fixed, because if the threshold is set low, more edges can be detected, and at the same time, it also brings a lot of noise and is easy to be interfered. If the threshold is set too high, some edges will be lost. Edge detection uses a threshold method with hysteresis to determine the threshold. Firstly, it uses the threshold to determine a starting point, and then tracks the edge path. If it is always greater than a certain limit, it is considered that the edge continues to track. If it is less than a certain value, it stops recording, which can eliminate some noise interference. Based on this, the purpose of setting detection and recognition mode is realized.

\section{EXPERIMENTAL TEST}

\section{A. Experimental preparation}

In order to test the effectiveness of the design technology, an experimental test is carried out. Experiments were performed using Manab 7.0. The experiment was configured as an Intel Core $2(1.6 \mathrm{GHz})$ processor with $1 \mathrm{G}$ memory and an operating system of Window10 Professional. The test video in this experiment is the video 
sequence shot by a CCD camera mounted on a car in Nanyang City. The parameters for the CCD camera are shown in Table 1.

Table 1. The parameters for the CCD camera

\begin{tabular}{cc}
\hline Index & Parameter \\
\hline Version & iKon-M912 \\
Interface & USB2.0 \\
Effective pixel & $320^{*} 240$ \\
Reading rate & $5 \mathrm{MHz}$ \\
Frame frequency & 15 Frames/Seconds \\
\hline
\end{tabular}

The camera erection height is about $1.4 \mathrm{~m}$, the pitch angle is 90.5 , which is in line with the erection height of the monitoring camera in the traffic system. The test video lasted 1 minute and 26 seconds, with a frame rate of 15 frames and a sampling rate of 24 bits. The entire video sequence was converted into 1300 images with 320 pixels in width and height of 240 pixels totaling $181 \mathrm{M}$ with image format suffix in bmp format. The received signal is simulated and processed by using MATLAB computer vision toolbox, and the moving car ahead is detected effectively. Using CCD conversion image sensor, the collected image information is converted into electrical signal, and then transmitted to the computer for processing. Under the above experimental preparation conditions, the experimental test is carried out and the experimental results are obtained.

\section{B. Experimental result}

In the experiment, the method proposed by Hakim and Zul [4] and the method proposed by Chen et al. [5] are selected and compared with the designed technology. Under complex motion conditions, the detection effects of three detection and recognition technologies are tested respectively. The more the number of vehicles detected from the same photo, the better the performance of the detection and identification technology of this method, because the more the number of vehicles identified, the method can accurately identify the characteristics of different motor vehicles and help to solve the daily management problem of traffic, and is of practical significance. The experimental results are shown in Figures 1-4. The black frame marks the identified vehicle.

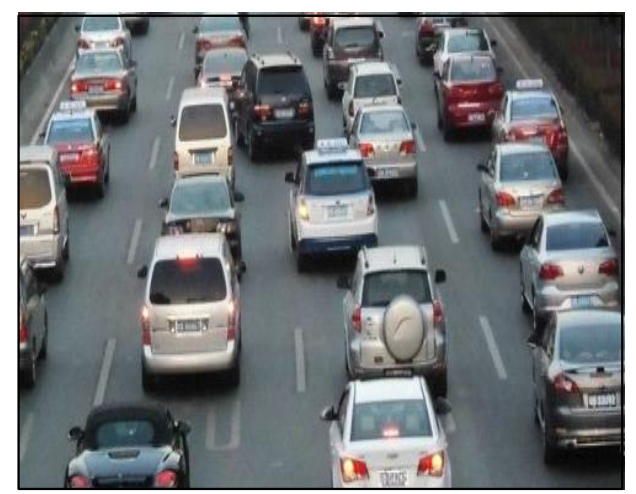

Figure 1. Image to be detected

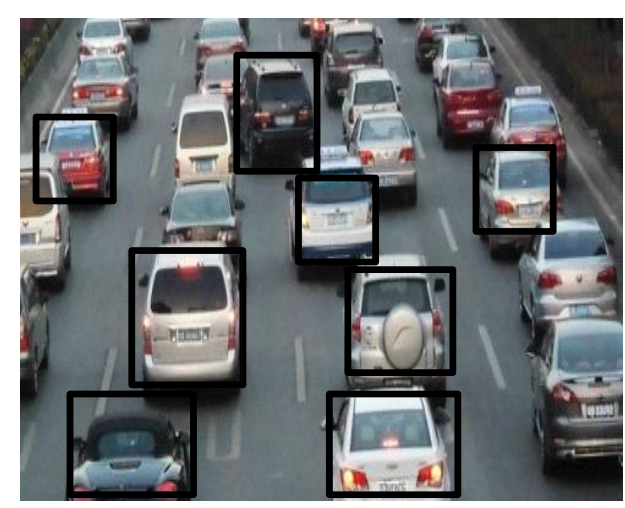

Figure 2. The designed technology detection image

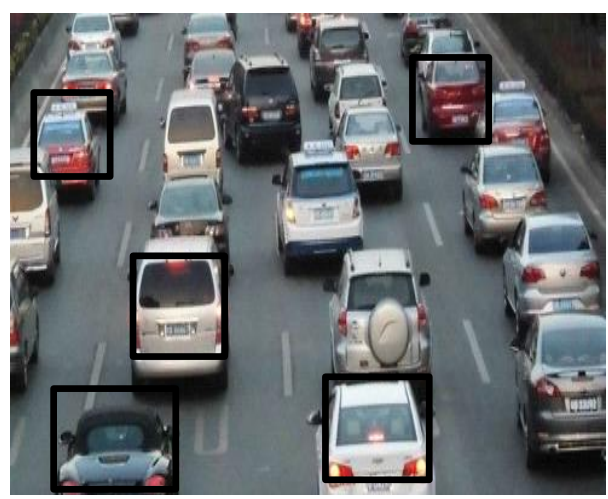

Figure 3. Image detection results of the method c

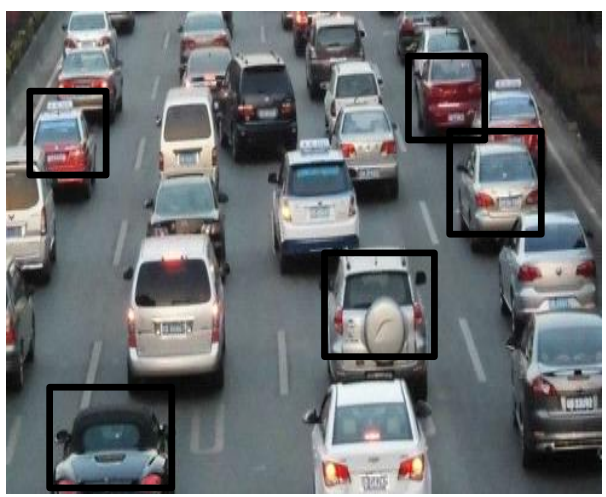

Figure 4. Image detection results of the method proposed by

Chen et al. [5]

The experimental results show that in the complex environment, when there are many cars in the moving scene, the mutual interference between cars is more serious, and there are more occlusion, the recognition and detection technology designed in this paper still has good detection and recognition effect. As shown from Figure 2, the designed motor vehicle detection and identification technology marks 8 vehicles, that is, 8 vehicles are identified, and on the basis of identifying the normal driving vehicle, the vehicle changing the lane can also be identified. As shown from Figures 3 and 4, the other two recognition detection technologies only detect 5 vehicles, which shows that the design of moving vehicle detection 
and recognition technology has more superior performance. This is because this method uses point operation to enhance the high-frequency information of the image, and first outline the video image tracking target, to lay the foundation for the later accurate identification. Artificial intelligence is used to extract the texture features of the moving vehicles, thus accurately identifying the target vehicle in complex images. Finally, the edge feature extraction algorithm was used to determine the detection effect and further improve the identification effect.

Further test the detection and identification efficiency of the three methods, the higher the detection efficiency, the better the detection performance. The results of the detection and identification time of the three methods are shown in Figure 5.

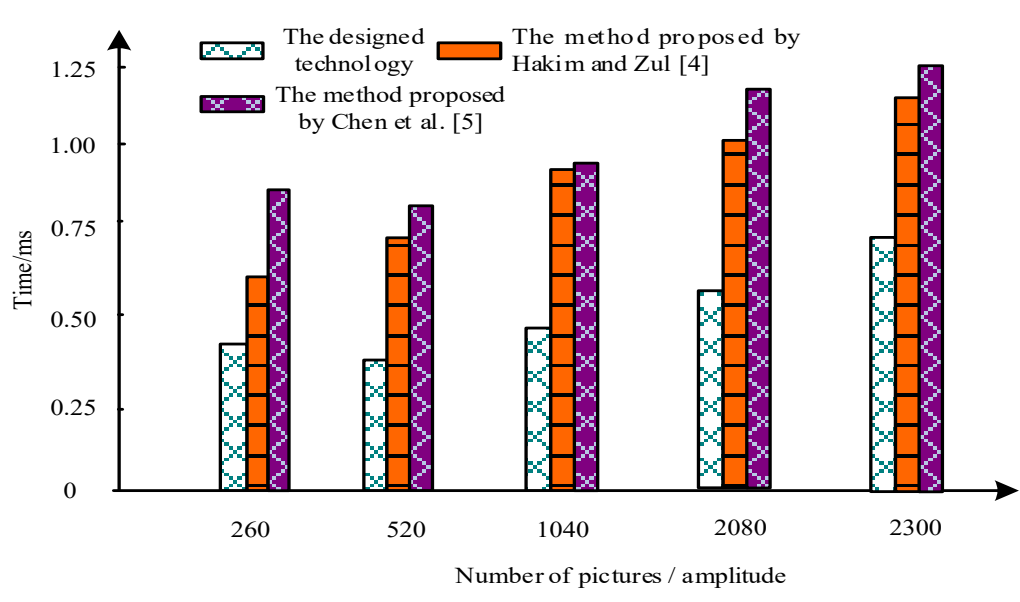

Figure 5. Time of detection and identification of the three methods

As can be seen from Figure 5, the present image recognition time of this method is less than $0.75 \mathrm{~ms}$, the recognition time is significantly less than the other two methods, and the identification efficiency is significantly higher than the other two methods, which proves the superior performance of the present method. The reason for the excellent results of this method is the ability to predict the motion target region under the motion vector similarity of the image, simplifying the complex process of real-time detection. The identification of the moving vehicle was accelerated by using the gray scale histogram distribution curve.

\section{CONCLUSIONS}

To improve the detection effect of motor vehicles, an artificial intelligence-based motor vehicle detection and recognition technology is designed. Point operation is used to improve the image contrast and depict the video image tracking target. The motor target region of the vehicle was predicted using the motor vector similarity. The AI method was used to extract the motor vehicle features, and the edge feature extraction algorithm was used to determine the detection and recognition patterns. The experimental results show that under complex conditions, the mobile vehicle detection and recognition technology designed here has a better monitoring function than the existing technology. It provides a new idea for the academic circles to carry out relevant research. At the same time, it broadens the application scope of artificial intelligence technology and makes the detection and recognition technology of moving vehicles more adaptable. Due to the limited research conditions of this paper, large computation and complex technical applications, the economic cost of this technology is high, and future research can reduce the complexity of the economic cost and ensure the identification effect.

\section{ACKNOWLEDGEMENT}

This work was supported by the Henan Science and Technology Project (No. 212102311007).

\section{REFERENCES}

[1] A. Dimri, H. Singh, N. Aggarwal, B. Raman, K. K. Ramakrishnan and D. Bansal, "Barosense: using barometer for road traffic congestion detection and path estimation with crowdsourcing". ACM Transactions on Sensor Networks, vol. 16, no. 1, pp. 1-24, 2019.

[2] X. Zhang, M. Zhou, P. Qiu, Y. Huang and J. Li, "Radar and vision fusion for the real-time obstacle detection and identification". Industrial Robot, vol. 46, no. 3, pp. 391-395, 2019.

[3] D. Roberts and M. Golparvar-Fare, "End-to-end vision-based detection, tracking and activity analysis of earthmoving equipment filmed at ground level". Automation in Construction, vol. 105, pp. 102811, 2019.

[4] L. Hakim and M. I. Zul, "Implementation of discrete wavelet transform on movement images and recognition by artificial neural network algorithm", WSEAS Transactions on Signal Processing, vol. 15, pp. 149-154, 2019.

[5] Y. M. Chen, M. Li and X. L. Fang, "Detection method for moving vehicle target based on fast RCNN speeded by spatial-temporal fusion", Electronic Measurement Technology, vol. 43, no. 3, pp. 7, 2020.

[6] A. Makkar, U. Ghosh and P. K. Sharma, "Artificial intelligence and edge computing-enabled web spam detection for next generation IOT applications", IEEE 
Sensors Journal, vol. 21, no. 22, pp. 25352-25361, 2021.

[7] Q. Li, M. G. Ji and J. Kim, "Grayscale nanopixel printing at sub-10-nanometer vertical resolution via light-controlled nanocapillarity", ACS Nano, vol. 14, no. 5, pp. 6058-6066, 2020.

[8] D. A. Suponnikov, A. Putilin, E. A. Tatarinova, Z. G. Zhgunev and A. R. Dabagov, "Development of an area image sensor pixel for an x-ray detector", Russian Microelectronics, vol. 48, no. 7, pp. 470-476, 2019.

[9] S. M. Jiao, M. J. Sun, Y. Gao, T. Lei, Z. W. Xie and X. C. Yuan, "Motion estimation and quality enhancement for a single image in dynamic single-pixel imaging", Optics Express, vol. 27, no. 9, pp. 12841-12854, 2019.

[10] W. Kazmi, I. Nabney, G. Vogiatzis, G., P. Rose and A. Codd, "An efficient industrial system for vehicle tyre (tire) detection and text recognition using deep learning", IEEE Transactions on Intelligent Transportation Systems, vol. 22, no. 2, pp. 1264-1275, 2020.

[11] S. Tumrani, Z. Y. Deng, H. Y. Lin and J. Shao, "Partial attention and multi-attribute learning for vehicle re-identification", Pattern Recognition Letters, vol. 138, pp. 290-297, 2020.

[12]I. S. Weon, S. G. Lee and J. K. Ryu, "Object recognition based interpolation with $3 \mathrm{~d}$ Lidar and vision for autonomous driving of an intelligent vehicle", IEEE Access, vol. 8, pp. 65599-65608, 2020.

[13] A. Sachu, S. Krishnendu, S. A. Sunny, G. K. Sudhi and M. P. Imthiyas, "Remarked vehicle detection and identification", International Journal of Computer Sciences and Engineering, vol. 7, no. 5, pp. 1633-1642, 2019.

[14]H. Yoo, H. J. Moon, S. H. Kim and S.-I. Choi, "Multi-target tracking with multiple 2D range scanners", IEEE Access, vol. 8, pp. 99990-99998, 2019.

[15] A. Appathurai, R. Sundarasekar, C, Raja, E. J. Alex, C. A. Palagan and A. Nithya, "An efficient optimal neural network-based moving vehicle detection in traffic video surveillance system", Circuits, Systems, and Signal Processing, vol. 39, no. 2, pp. 734-756, 2020.

[16] V. Chand and J. Karthikeyan, "RFID-GPS based mechanism using AODVRP for stolen vehicle detection in vanet", Indonesian Journal of Electrical Engineering and Computer Science, vol. 18, no. 1, pp. 396-404, 2020.

[17] Y. Y. Kong, B. W. Zhang, Y. Y. Chen, H. Leung and S. Y. Xi, "3-D point cloud reconstruction of infrared images based on improved structure from motion", WSEAS Transactions on Signal Processing, vol. 15, pp. 14-19, 2019.

[18] M. A. Rao, F. S. Khan and J. Laaksonen, "Compact deep color features for remote sensing scene classification", Neural Processing Letters, vol. 53, no. 2, pp. 1523-1544, 2021

[19]F. Damayanti, W. K. Dewi, E. Rahmanita and A. R. Rachmad, "Detection and identification Indonesia license plate using background subtraction based on area", Journal of Physics: Conference Series, vol. 1569, no. 2, pp. 022064, 2020.

[20] V. Priyashman and W. Ismail, "Signal strength and read rate prediction modeling using machine learning algorithms for vehicular access control and identification", IEEE Sensors Journal, vol. 19, no. 4, pp. 1400-1411, 2019.

[21] K. F. Zhu, J. G. Wang and W. Q. Ye, "Low-resolution radar target recognition algorithm with unbalanced samples", Computer Simulation, vol. 38, no. 3, pp. 10-14, 185, 2021.

[22] A. Bouchet, P. Alonso, I. Diaz and S. Montes, "On the performance of some edge detectors for gray scale images", Journal of Multiple-Valued Logic and Soft Computing, vol. 32, no. 3-4, pp. 241-256, 2019.

[23] R. Dhivya and R. Prakash, "Edge detection of satellite image using fuzzy logic", Cluster Computing, vol. 22, no. 1, pp. 1-8, 2019.

\section{Creative Commons Attribution License 4.0 (Attribution 4.0 International, CC BY 4.0)}

This article is published under the terms of the Creative Commons Attribution License 4.0

https://creativecommons.org/licenses/by/4.0/deed.en_US 